\title{
El jurel Trachurus murphyi y las variables ambientales de macroescala
}

\author{
The Jack mackerel Trachurus murphyi and the environmental macro-scale variables
}

Marco Espino

Instituto del Mar del Perú, esquina Gamarra y General Valle S/N Chucuito, Callao, Perú.

Email Marco Espino: mespino@imarpe.gob.pe
Citación:

Espino M. 2013. El jurel Trachurus murphyi y las variables ambientales de macroescala. En: Csirke J., R. GuevaraCarrasco \& M. Espino (Eds.). Ecología, pesquería y Carrasco \& M. Espino (Eds.). Ecologia, pesquería y
conservación del jurel (Trachurus murphyi) en el Perú. Rev. peru. biol. número especial 20(1): 009- 020 (Septiembre 2013)

\section{Resumen}

Se analiza información de diversas variables ambientales de macroescala disponibles desde 1876 para el Pacífico Sudoriental y datos más recientes de desembarques y biomasas de jurel Trachurus murphyi Nichols 1920 en el mar peruano en relación con áreas probables de masas de agua equivalentes a Aguas Costeras Frías (ACF) y Aguas Subtropicales Superficiales (ASS). Se concluye que el Índice de Oscilación Decadal del Pacífico (PDO) presenta expresiones de variabilidad coherentes con las encontradas para el Índice de Oscilación del Sur (SOI) y que los cambios observados en las biomasas de T. murphyi en el mar peruano reflejan cambios en la disponibilidad del recurso asociado a patrones de variabilidad seculares (SOI) y decadales (PDO). Tales fluctuaciones en la disponibilidad del recurso afectan las pesquerías de Ecuador, Perú y norte de Chile, las que muestran variaciones importantes en sus desembarques y habrían dado una imagen sesgada de la abundancia conduciendo a diagnósticos errados de la real situación del recurso. Estos patrones de variabilidad afectarían también las manifestaciones de El Niño, haciendo que se inicien en otoños o primaveras australes dependiendo de si el PDO imperante es negativo o positivo. Por otro lado, en función a la distancia euclidiana de las varianzas del SOI se identifican periodos de alta (1876 - 1925 y 1976 - 2012) y baja (1926 - 1975) variabilidad; y con el PDO se diferencian periodos cálidos (1925 - 1944 y 1975 - 1994), frio (1945 - 1974) y templados o interfaces (1895 - 1924 y 1995 - 2012), siendo estos últimos producto de la interacción con los momentos de alta variabilidad.

Palabras clave: Jurel, variabilidad ambiental, PDO, SOI, distancia euclidiana

\section{Abstract}

This paper analyses information on various macro environmental variables available since 1876 for the Southeast Pacific and more recent data on Jack mackerel Trachurus murphyi (Nichols, 1920) landings and biomass in the Peruvian sea, relating them to probable areas of water masses equivalent to Cold Coastal Waters (CCW) and Subtropical Surface Waters (SSW). It is concluded that the index of the Pacific Decadal Oscillation (PDO) presents expressions of variability that are consistent with those found for the Southern Oscillation Index (SOI) and that the detected changes in biomass of Jack mackerel T. murphyi in the Peruvian sea reflect changes in the availability of the fish stock associated with secular (SOI) and decadal (PDO) variability patterns. These fluctuations in stock availability impact fisheries in Ecuador, Peru and northern Chile, which show significant variations in their landings and would have given a biased picture of the state of abundance, leading to wrong diagnoses of the real situation of the exploited stocks. These patterns of variability would also affect the appearance of EI Niño, making them start in the southern hemisphere autumn or spring depending on whether the current PDO is positive or negative. Periods of high (1876 - 1925 and 1976 - 2012) and low (1926 - 1975) variability are also identified in relation to the Euclidean distance of the variances of the SOI; and in relation to the PDO a distinction is made between warm (1925 - 1944 and 1975 - 1994), cold (1945 - 1974) and tempered or interface periods (1895 - 1924 and 1995 - 2012), the latter being explained by the interaction between periods of high variability.

Keywords: Jack mackerel, environmental variability, PDO, SOI, Euclidian distance. 


\section{Introducción}

El Pacífico Sudoriental está sometido permanentemente a procesos de variabilidad ambiental que afectan los principales aspectos de la dinámica poblacional de los recursos pesqueros, como el reclutamiento, crecimiento, mortalidad natural, mortalidad por pesca (capturabilidad) y los patrones de comportamiento. Estos efectos se manifiestan a escala, intensidad y frecuencia diferentes, condicionando respuestas que finalmente constituyen estados de la misma población frente a la combinación de los factores de variabilidad correspondientes a cada uno de los momentos o periodos ambientales prevalentes, y que como ya se ha observado pueden ser de corto (estacionales), mediano (interanuales-ENSO), largo plazo (decadales) y muy largo plazo (seculares) (Espino 2003, Espino \& Yamashiro 2012).

En este trabajo se presenta un análisis de algunos factores de la variabilidad ambiental a macroescala, en búsqueda de regularidades o patrones normativos ambientales de variabilidad (Espino 2003). Los que se plantean a través de modelos conceptuales que permiten caracterizar e identificar escenarios ambientales que explican cambios observados en las variables biológico-pesqueras del jurel Trachurus murphyi Nichols 1920 y su entorno ambiental, propios del mar peruano.

De lograrse, ello contribuiría a plantear acciones de manejo acordes con cada momento o escenario ambiental y el probable estado poblacional coherente con dicho momento. En este contexto, la planificación de la producción pesquera en el corto, mediano y largo plazo podría contribuir a evitar cambios socioeconómicos y financieros traumáticos, y también favorecería el mejor aprovechamiento del recurso en cada estado y en particular en momentos de bonanza causados por el advenimiento de condiciones poblacionales favorables, bajo escenarios ambientales propicios para esta especie.

\section{Material y métodos}

Los datos mensuales del Índice de Oscilación del Sur (SOI, Southern Oscillation Index) de 1876 a 2012 fueron obtenidos del Bureau of Meteorology, National Climate Center of Australia. Basándose en la oscilación armónica definida de acuerdo a la siguiente expresión:

$\operatorname{Var}$ IOS $=7.5-1.5[\operatorname{sen}(2 * 3.1416 \mathrm{Ti}-2050+5) / 100]$ (Espino, 2003)

Mediante la cual se plantea un modelo conceptual en el que se diferencian periodos o momentos de alta y baja variabilidad para el Pacífico Sur, en función a las desviaciones estándar del SOI. También se promediaron las desviaciones estándar anuales del SOI cada 50 ańos (1876 - 1925, 1926 - 1975 y 1976 - 2012) con lo cual se obtuvieron agrupamientos por distancia euclidiana.

Los datos mensuales del Índice de Oscilación Decadal del Pacífico (PDO, del inglés Pacific Decadal Oscillation) de 1900 a 2012 fueron obtenidos del Joint Institute for the Study of the Atmosphere and Ocean (JISAO), con los cuales Espino (2003) determinó una oscilación armónica asimétrica que responde a un modelo conceptual de acuerdo a la siguiente expresión:

$\mathrm{PDO}=-0.25-1.5[\operatorname{sen}(2 * 3.1416 \mathrm{Ti}-1880+5) / 50$ (Espino 2003)

Siguiendo los arreglos: 1895 - 1924, 1925 - 1944, 1945 1974, 1975 - 1994 y 1995 - 2012, se promediaron los datos anuales de PDO, para luego definir agrupamientos térmicos diferenciados por distancia euclidiana.

Entre 1950 y 2012, los datos mensuales del Índice Multivariado ENSO (MEI, Multivariate ENSO Index) se obtuvieron del Earth System Research Laboratory de la NOAA y los datos mensuales de temperatura superficial del mar (TSM) de Chicama fueron obtenidos de la base de datos institucional del IMARPE.

De acuerdo a la serie disponible de las variables ambientales MEI y TSM-Chicama, se promediaron por cuarto de siglo (1925 - 1949, 1950 - 1974, 1974 - 1999 y 2000 - 2012), los cuales se agruparon por distancia euclidiana para definir escenarios ambientales térmicos.

Las áreas de las isotermas $\left(13\right.$ a $\left.28^{\circ} \mathrm{C}\right)$ fueron proporcionadas por la Unidad de Percepción Remota y Sistemas de Información Geográfica (UPRSIG) del IMARPE y correspondieron a promedios anuales del espacio geográfico comprendido entre $\operatorname{los} 02^{\circ} \mathrm{N}-20^{\circ} \mathrm{S}$ y los $85-68^{\circ} \mathrm{W}$. En el periodo $1982-2012$, los tamaños promedio anuales de las áreas de cada isoterma (13 a $\left.28{ }^{\circ} \mathrm{C}\right)$ se correlacionaron una a una $(13 / 14,14 / 15,15 / 16$, $16 / 17,17 / 18,18 / 19,19 / 20,20 / 21,21 / 22,22 / 23,23 / 24,24 / 25$, 25/26, 26/27 y 27/28), obteniéndose coeficientes de correlación de Pearson (r), a partir de los cuales se planteo una propuesta operacional de identificación de masas de aguas superficiales, mediante el levantamiento de cartas satelitales de TSM. Además, se calcularon valores de TSM Medias Ponderadas (TSMP) al área promedio anual de las isotermas correspondientes, de acuerdo a la siguiente formulación:

TSMP $=\Sigma\left(\right.$ To isoterma*Área $\left(\mathrm{km}^{2}\right)$ isoterma $) /(\Sigma$ Áreas de las isotermas)

De ésta se derivaron las anomalías de la TSMP (ATSM) según la siguiente ecuación:

\section{ATSM=TSMP(i) - Promedio de la TSMP(1982 - 2012)}

Se analizaron los desembarques anuales de 1960 a 2012, obtenidos del Ministerio de la Producción del Perú, de doce especies de peces que representan en promedio el $96.5 \%$ de los desembarques nacionales, los cuales fueron estandarizados para cada ańo de acuerdo a lo propuesto por Noakes y Beamish (2009).

Las especies seleccionadas fueron el jurel T. murphyi, anchoveta Engraulis ringens, sardina Sardinops sagax, caballa Scomber japonicus, bonito Sarda chiliensis chiliensis, merluza Merluccius gayi peruanus, cojinova Seriolella violacea, pejerrey Odontesthes regia, cavinza Isacia conceptionis, machete Ethmidium maculatum, sierra Scomberomorus sierra y la pota o jibia Dosidicus gigas.

Se establecieron relaciones entre los desembarques de $T$. murphyi con los de las otras especies, para lo cual se empleó la correlación de Pearson (r) para arreglos decadales (décadas de los sesenta, setenta, ochenta, noventa, y el periodo comprendido entre el 2000 y el 2010). Por otro lado, las biomasas T. murphyi fueron relacionadas con las variables ambientales también bajo los mismos arreglos decadales.

Los análisis de agrupamiento fueron realizados por distancia euclidiana y análisis de similitud (similaridad) de Bray Curtis, empleando el programa PRIMER 6.0.

\section{Resultados}


Variables ambientales de macroescala espacial.- El análisis de mediano y largo plazo de las variables ambientales de macroescala permitiría explicar los patrones de distribución de T. murphyi en el mar peruano y en el Pacífico Sudoriental en general. El efecto de estas variables en la dinámica poblacional podría apreciarse a partir del mejor conocimiento de las mismas.

El Índice de Oscilación del Sur (SOI).- Desde 1876 la serie disponible del SOI refleja una alta variabilidad en procesos estacionales (verano-invierno), interanuales (El Niño-La Niña), decadales (décadas cálidas-décadas frías) y seculares (alta y baja variabilidad). Es así que entre 1876 y 1925 se presentan valores más extremos, negativos en los años 1877, 1888, 1896, 1905 y positivos en $1879,1910,1917$, reflejando una mayor variabilidad o relativa inestabilidad. Entre 1925 y 1975, las manifestaciones extremas negativas $(1940,1941)$ y positivas (1950) fueron menos frecuentes y menos intensas, lo que expresó un patrón de menor variabilidad o relativa estabilidad. Luego, desde 1976 a la actualidad se repite una tendencia similar a la observada entre 1876 y 1925 (Figs. 1 y 2).

Un análisis de agrupamiento utilizando la distancia euclidiana evidencia la separación de esos periodos (Fig. 2). De acuerdo a ello se identifican momentos de alta y baja variabilidad en el Pacífico Sudoriental, expresados en manifestaciones más y menos intensas de El Niño y La Niña, respectivamente.

Los momentos de alta variabilidad con expresiones máximas o muy fuertes de El Niño se produjeron en los primeros y últimos cuartos de cada siglo coincidentemente con lo que planteó la serie de Quinn et al. (1987) donde las expresiones "muy fuertes" (VS: Very Strong) de El Niño se observaron en los años 1578, 1728, 1791, 1828, 1877-78, 1891, 1925-26, 1982-83 y $1997-$ 98. Obsérvese que tanto en el siglo XIX como en el siglo XX se presentaron tres manifestaciones extremas de El Niño, dos de las cuales se observaron en el último cuarto de los referidos siglos y una en el comienzo (1925).

Probablemente, en los siglos anteriores ocurrieron manifestaciones similares a las acaecidas en el siglo XX, pero no pueden ser definidas claramente, porque la información utilizada esta basada en reportes de cronistas e historiadores sobre los impac-

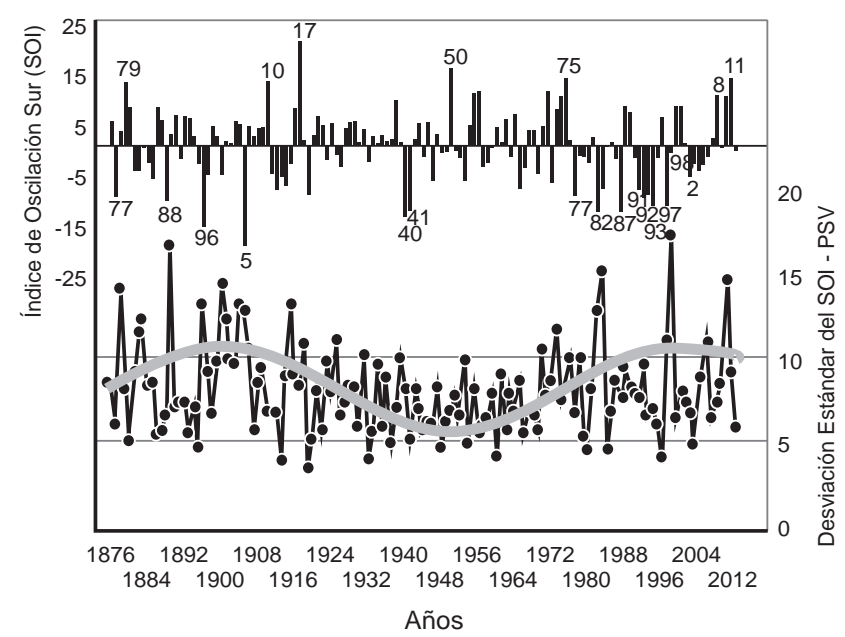

Figura 1. Índice de Oscilación del Sur (SOI) y su varianza expresada como Índice de Variabilidad Secular del Pacífico (PSV) desde 1876 al 2012.

Figure 1. Southern Oscillation Index (SOI) and its variance expressed as Pacific Secular Variability Index (PSV) from 1876 to 2012. tos del evento en el ambiente, en la pesquería y en la vida de las aves y mamíferos marinos, que podrían estar distorsionados por la apreciación subjetiva de los autores de tales registros. Sin embargo, a pesar de ello no dejan de ser señales útiles para el análisis de las manifestaciones extremas de El Niño.

Quinn et al. (1987) reportaron manifestaciones de El Niño desde 1525, observándose que eventos muy fuertes no han sido registrados más de tres en cada siglo. Podemos destacar el siglo XVII en que no se produjo ninguna manifestación de tal magnitud (Tablas 1 y 2), esto es coincidente con la pequeña edad glacial del medioevo, cuando se produjo el Mínimo de Maunder (MM), caracterizado por extremas temperaturas bajas que afectaron a grandes áreas del planeta (Maenza \& Compagnucci 2010).

Por su parte, en los cuartos de siglo intermedios (1925 1975), de menor variabilidad, las manifestaciones de El Niño son menos frecuentes y de menor intensidad, no mostrando manifestaciones fuertes. Tal es el caso de los años 1940 y 1941, en los que la magnitud (SOI) del fenómeno fue significativa (fuerte), pero como expresión de variabilidad no lo fue, pues su desviación estándar no fue importante, con valores medios anuales de -13.8 y -12.9 , pero con una baja desviación estándar.

En conclusión, los datos expresan que en este periodo, en intervalos alternados de aproximadamente 50 ańos, el SOI y su varianza definen momentos de alta y baja variabilidad, con manifestaciones fuertes y muy fuertes de El Niño y La Niña, en los últimos y primeros cuartos de cada siglo. En los cuartos de siglo intermedios, son menos probables las manifestaciones severas de este fenómeno. Estos momentos con dominancia de SOI positivos aumentarían la probabilidad de mayor disponibilidad del stock norte (far north) del jurel del Pacífico Sudoriental.

El patrón de variabilidad expresado por los valores anuales altos y bajos de la desviación estándar del SOI se ha denominado Variabilidad Secular del Pacífico (PSV) (Espino 2003), por corresponder a ciclos armónicos simétricos completos de 100 años, que significan una expresión de intensidad de variabilidad en ambos extremos: positivo y negativo. Sin embargo, este patrón no constituye manifestación térmica de variabilidad como el PDO.

La Oscilación Decadal del Pacífico (PDO).- La Oscilación Decadal del Pacífico (PDO) (Hare 1996, Mantua et al. 1997) es un patrón de variabilidad climática espacial persistente $(20 \mathrm{a}$ 30 años), que abarca todo el Océano Pacífico (Fig. 3) con ma-

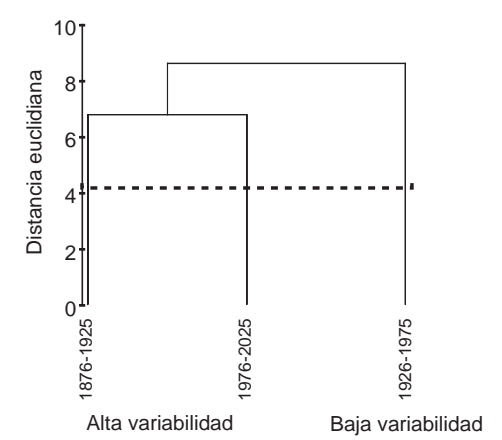

Figura 2. Distancia euclidiana aplicada a la desviación estándar del SOI, que agrupa los periodos de alta variabilidad (1876 - 1925 y 1976 - 2012) diferenciándolos de los de baja variabilidad (1926 - 1975).

Figure 2. Euclidean distance applied to the standard deviation of the SOI, which groups high variability periods (1876 - 1925 and 1976 2011 ) distinguishing them from those of low variability (1926 - 1975). 
nifestaciones muy claras en el Pacífico Norte (Norteamérica) y efectos secundarios en los trópicos. Todo lo contrario de lo que sucede con el proceso ENSO, cuyo escenario es la zona intertropical. Mantua (1999a y b) describe manifestaciones de este evento entre 1890 y 1924 (fase fría), entre 1925 y 1946 (fase cálida), entre 1947 y 1976 (fase fría) y, finalmente, de 1977 hasta mediados de la década de los noventa (fase cálida). Observando la Figura 3 se puede pronosticar que en la segunda mitad de la década de los noventa se habría iniciado una fase fría (Espino \& Yamashiro 2012).

En esa medida, para la zona ecuatorial, también se definen fases positivas y negativas del PDO, que se alternan entre sí condicionando escenarios fríos y cálidos para la zona norte del Pacífico Sudoriental, es decir, en el mar peruano y ecuatoriano. Durante el primer cuarto del siglo XX, se alternan ańos cálidos y fríos, lo cual coincide con un periodo de alta variabilidad expresada en la varianza del SOI (Fig. 1). Luego, se observa una fase positiva que se extiende hasta 1946. Entre 1946 y 1975 se observan valores negativos del PDO, con 5 manifestaciones positivas (1948, 1958, 1960, 1961 y 1970), lo que representa sólo el 17\% de la serie. Es decir, entre 1926 y 1975, se puede observar que está claramente definido que a una fase cálida le sigue una fría, lo cual coincide con un periodo de baja variabilidad del SOI. De 1976 a 1995 predominaron condiciones positivas con sólo 4 desviaciones negativas (1989, 1990, 1991 y 1995) que representan el 20\% de la serie. Luego, entre 1996 y 2012 se alternan desviaciones positivas y negativas, con comportamiento similar al observado previamente a 1925.

El análisis de agrupamiento por distancia euclidiana entre periodos diferenciados del PDO determina tres grupos de años que corresponden a periodos cálidos (predominio de anomalías positivas), periodos fríos (predominio de anomalías negativas) y periodos intermedios o de interfase que, en adelante, se denominarán periodos templados porque en tales momentos se intercalan desviaciones positivas y negativas con la misma frecuencia (9) hasta el 2012 (Fig. 4). Esto sugiere que en las

Tabla 1. Eventos El Niño fuertes y muy fuertes acaecidos en el Perú desde 1525 a la actualidad (actualizado después de Quinn et al. 1987) Table 1. Strong and very strong EI Niño events occurred in Peru since 1525 to date (updated after Quinn et al. 1987)

\begin{tabular}{|c|c|c|c|c|c|}
\hline Año & Int & Año & Int & Año & Int \\
\hline $1525-1526$ & $S$ & $1687-1688$ & $\mathrm{~S}+$ & 1871 & S \\
\hline $1531-1532$ & $S$ & 1696 & $S$ & $1877-1878$ & VS \\
\hline $1539-1541$ & $S$ & 1701 & $\mathrm{~S}+$ & 1884 & $\mathrm{~S}+$ \\
\hline 1552 & S & $1707-1708$ & S & 1891 & VS \\
\hline $1567-1568$ & S+ & $1714-1715$ & $S$ & $1899-1900$ & S \\
\hline 1574 & $S$ & 1720 & $\mathrm{~S}+$ & $1911-1912$ & $S$ \\
\hline 1578 & VS & 1728 & VS & 1917 & S \\
\hline $1591-1592$ & S & 1747 & S & $1925-1926$ & VS \\
\hline 1607 & $S$ & 1761 & $S$ & 1932 & $S$ \\
\hline 1614 & $S$ & 1775 & $S$ & 1940 - 1941 & S \\
\hline $1618-1619$ & $S$ & 1785 - 1786 & S & $1957-1958$ & $S$ \\
\hline 1624 & S & 1791 & VS & $1972-1973$ & S \\
\hline 1634 & S & $1803-1804$ & S+ & $1982-1983$ & VS \\
\hline 1652 & $\mathrm{~S}+$ & 1814 & $S$ & $1997-1998$ & VS \\
\hline 1660 & S & 1828 & VS & $2009-2010$ & $S$ \\
\hline 1671 & $S$ & $1844-1845$ & S+ & & \\
\hline 1681 & S & 1864 & S & & \\
\hline
\end{tabular}

$\overline{\text { Int = Intensidad; S = Fuerte (Strong); VS = Muy fuerte (Very Strong) }}$

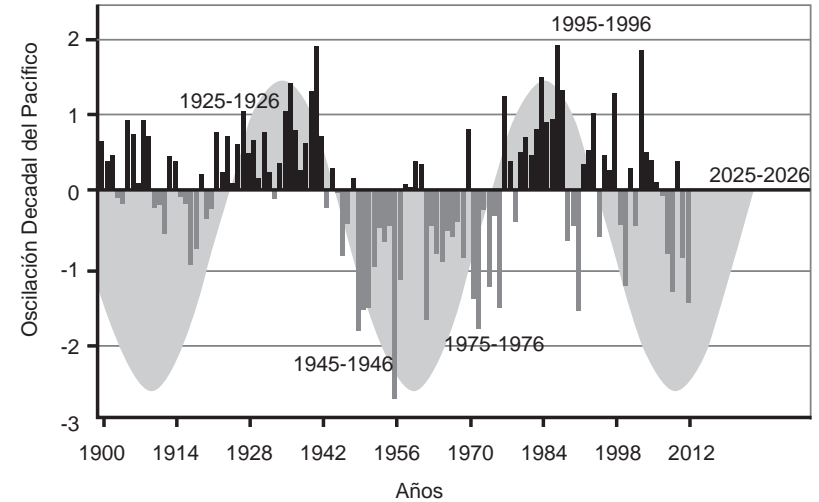

Figura 3. Oscilación Decadal del Pacífico (1900 - 2012).

Figure 3. Pacific Decadal Oscillation (1900 - 2012).

fases de SOI de alta variabilidad se producen recurrentemente desviaciones en ambos sentidos, lo cual imposibilita la definición clara de un estado cálido o frío, según corresponda, mientras que las manifestaciones del PDO son más evidentes en su estado positivo o negativo en las fases de baja variabilidad.

Se puede concluir que el PDO presenta expresiones de variabilidad coherentes con las encontradas para el SOI. Entre estas manifestaciones, en la fase intermedia (1926 - 1975) de baja variabilidad del SOI, se destaca la definición clara de un periodo cálido (entre 1925 y 1944) y un periodo frío (entre 1945 y 1974), mientras que los denominados periodos templados se diferencian claramente en los momentos de alta variabilidad, debido a que en

Tabla 2. Eventos EI Niño casi moderados, moderados, fuertes y muy fuertes acaecidos en el Perú desde 1803 a la actualidad (Actualizado de Quinn et al. 1987).

Table 2. Almost moderate, moderate, strong and very strong EI Niño events in Peru occurred since 1803 to date (Updated from Quinn et al. 1987)

\begin{tabular}{cc|cc|cc}
\hline Año & Int & Año & Int & Año & Int \\
\hline $1803-1804$ & S+ & $1867-1868$ & $\mathrm{M}$ & $1925-1926$ & $\mathrm{VS}$ \\
$1806-1807$ & $\mathrm{M}$ & 1871 & $\mathrm{~S}+$ & $1930-1931$ & $\mathrm{~W} / \mathrm{M}$ \\
1812 & $\mathrm{M}$ & 1874 & $\mathrm{M}$ & 1932 & $\mathrm{~S}$ \\
1814 & $\mathrm{~S}$ & $1877-1878$ & $\mathrm{VS}$ & 1939 & $\mathrm{M}+$ \\
1817 & $\mathrm{M}+$ & 1880 & $\mathrm{M}$ & $1940-1941$ & $\mathrm{~S}$ \\
1819 & $\mathrm{M}+$ & 1884 & $\mathrm{~S}+$ & 1943 & $\mathrm{M}+$ \\
1821 & $\mathrm{M}$ & $1887-1889$ & $\mathrm{~W} / \mathrm{M}$ & 1951 & $\mathrm{~W} / \mathrm{M}$ \\
1824 & $\mathrm{M}$ & 1891 & $\mathrm{VS}$ & 1953 & $\mathrm{M}+$ \\
1828 & $\mathrm{VS}$ & $1896-1897$ & $\mathrm{M}+$ & $1957-1958$ & $\mathrm{~S}$ \\
1832 & $\mathrm{M}$ & $1899-1900$ & $\mathrm{~S}$ & 1965 & $\mathrm{M}+$ \\
1837 & $\mathrm{M}$ & 1902 & $\mathrm{M}+$ & $1972-1973$ & $\mathrm{~S}$ \\
$1844-1845$ & $\mathrm{~S}+$ & 1905 & $\mathrm{~W} / \mathrm{M}$ & 1976 & $\mathrm{M}$ \\
1850 & $\mathrm{M}$ & 1907 & $\mathrm{M}$ & $1982-1983$ & $\mathrm{VS}$ \\
1854 & $\mathrm{~W} / \mathrm{M}$ & $1911-1912$ & $\mathrm{~S}$ & 1987 & $\mathrm{M}$ \\
$1857-1858$ & $\mathrm{M}+$ & 1914 & $\mathrm{M}+$ & $1991-1994$ & $\mathrm{M}$ \\
1860 & $\mathrm{M}$ & 1917 & $\mathrm{~S}$ & $1997-1998$ & $\mathrm{VS}$ \\
1864 & $\mathrm{~S}$ & $1918-1919$ & $\mathrm{~W} / \mathrm{M}$ & 2002 & $\mathrm{M}$ \\
1866 & $\mathrm{M}$ & 1923 & $\mathrm{M}$ & $2009-2010$ & $\mathrm{~S}$ \\
\hline
\end{tabular}

Int = Intensidad; $\mathrm{W} / \mathrm{S}=$ Casi moderado (Near Moderate); $\mathrm{M}=$ Moderado (Moderate) S = Fuerte (Strong); VS = Muy fuerte (Very Strong) 


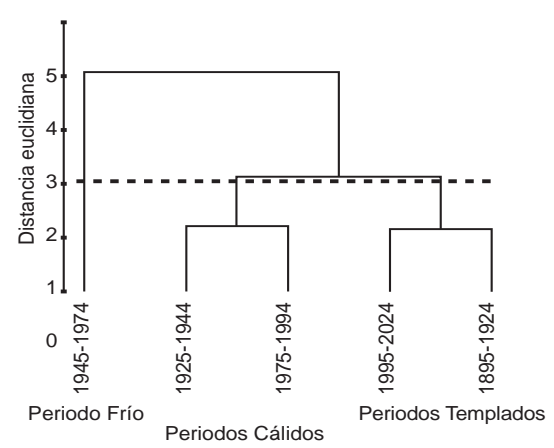

Figura 4. Distancia euclidiana aplicada al PDO diferenciando tres agrupamientos térmicos correspondientes a periodos cálidos (1925 - 1944 y 1975 - 1994), templados o interfases (1895 - 1924 y 1995 -2024) y frío $(1945-1974)$.

Figure 4. Euclidean distance applied to the PDO distinguishing three thermal groupings, corresponding to warm (1925 - 1944 and 1975 1994), temperate or interface (1895 - 1924 y $1995-2024)$ and cold (1945 - 1974) periods.

ellos se alternan desviaciones positivas y negativas, que responden al patrón de alta variabilidad imperante. Al respecto, de 1995 a 2012, hemos tenido 9 desviaciones positivas y 9 negativas, lo que coincide con lo observado el siglo pasado. Es de esperar que esta dinámica persista en tanto dure el periodo de alta variabilidad que atravesamos $(1976$ - 2025) y que, luego de concluido, se den condiciones similares a las obtenidas a mediados del siglo XX, repitiéndose una fase de baja variabilidad (2026 - 2075) (Figs. 2, 3 y 4).

Respecto al jurel, esta especie estaría más disponible a la pesquería de Perú y Ecuador cuando predominen valores positivos del PDO, tal como lo que se observó entre finales de la década de los setenta e inicios de los noventa, así como recientemente cuando han predominado condiciones cálidas (2003 - 2006). Se puede pronosticar que en los próximos años (al 2025) habrá desviaciones alternadas, negativas y positivas del PDO, las cuales alejarán o acercarán a T. murphyi de las costas del norte de Chile, Perú y Ecuador, lo que dependerá del periodo del SOI que atravesemos. Luego, tendremos el advenimiento de un persistente PDO positivo (+) cuando las condiciones sean manifiestamente favorables para aumentar la disponibilidad del stock norte ( far north) del jurel T. murphyi del Pacífico Sudoriental.

Índice Multivariado ENSO (El Niño Southern Oscillation).- El Índice Multivariado ENSO (MEI) (Wolter 1987, Wolter \& Timlin 1993, 1998) es el mejor indicador para el monitoreo de El Niño debido a que integra seis variables que reflejan el acoplamiento entre el océano y la atmósfera, siendo menos sensible a las variaciones ocasionales, por efectos locales de algunas variables. El MEI integra la presión a nivel del mar $(\mathrm{P})$, componentes zonales $(\mathrm{U})$ y meridionales $(\mathrm{V})$ del viento superficial, temperatura superficial del mar $(S)$, temperatura superficial del aire (A) y cobertura total del cielo (C).

En la serie disponible entre 1950 y el 2011, se revelan dos escenarios ambientales: uno con dominancia de valores negativos, entre 1950 y 1976; y un segundo, entre 1977 y 2006, con dominancia de valores positivos. Luego, son recurrentes las desviaciones negativas (Fig. 5) en los últimos cinco años. Se podría concluir que éstos corresponderían a escenarios ambientales fríos y cálidos, respectivamente. El primero de ellos coincide con la declinación

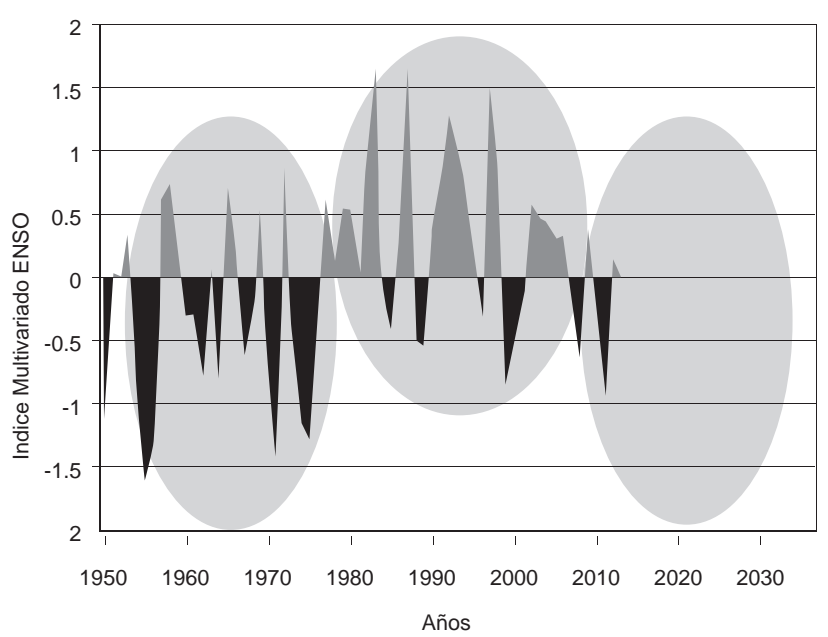

Figura 5. Índice multivariado ENSO - El Niño Southern Oscillation (1950 - 2012).

Figure 5. Multivariate ENSO Index (1950 - 2012).

de una fase de baja variabilidad y con una fase fría del PDO, mientras que el segundo coincide con el inicio de una fase de alta variabilidad y con una fase cálida del PDO. Asimismo, debemos considerar que además de ser muy útil para monitorear El Niño, el MEI también es de utilidad para definir escenarios ambientales como sucede con el SOI y TSM de Chicama.

Ahora bien, si se analiza la serie del Índice Multivariado ENSO por cuartos de siglo, por distancia euclidiana se obtiene dos agrupamientos que reflejan dos periodos fríos (1950-1974 y 2000 - 2012) y uno cálido (1975 - 1999) (Figs.6 y 7).

Temperatura superficial del mar (TSM) y sus anomalías (ATSM).- En el Perú y el Pacífico Sudoriental, la serie más amplia de información (1925 - 2012) de Temperatura Superficial del Mar (TSM) y sus anomalías (ATSM) es la que corresponde a la estación costera de Chicama (0741'41”S - 79²6’03”W). Esta serie revela que la variabilidad es una característica en el Ecosistema de Afloramiento Peruano (EAP). Es decir, en el mar peruano la "normalidad" es la variabilidad (Espino \& Yamashiro 2012). En esa medida, debemos considerar que en el Pacífico Sudoriental oscilan anomalías positivas y negativas, cuyas

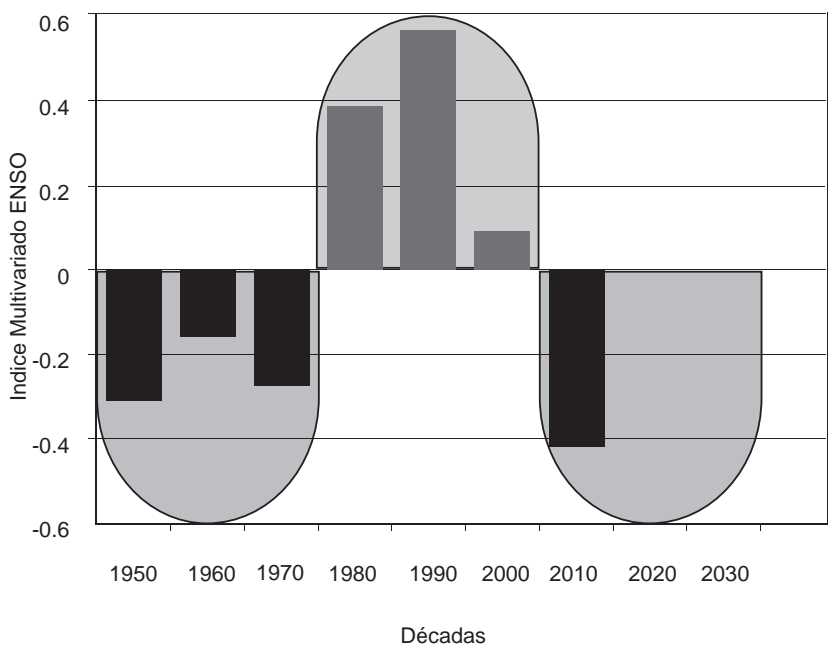

Figura 6. Índice Multivariado ENSO por décadas (1950 - 2010).

Figure 6. Multivariate ENSO Index by decades (1950 - 2010). 


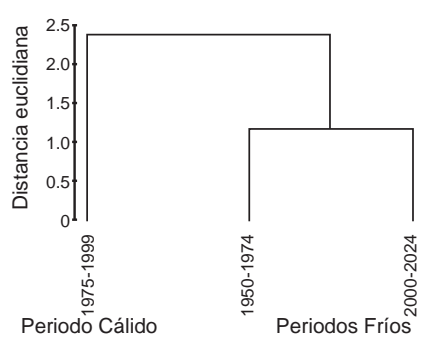

Figura 7. Distancia euclidiana entre valores promedio por cuartos de siglo del Índice Multivariado ENSO (1950 - 2012).

Figure 7. Euclidean distance between quarter - century average values of the Multivariate ENSO Index (1950 - 2012).

manifestaciones extremas corresponden a El Niño y La Niña. Las anomalías positivas son menos frecuentes, pero de mayor intensidad que las negativas. Las anomalías negativas muestran mínimas anuales promedio de $2{ }^{\circ} \mathrm{C}$, en tanto que las anomalías positivas alcanzan un promedio anual de $4.5^{\circ} \mathrm{C}$. Mensualmente, las anomalías positivas pueden llegar a +10 y las negativas a -3.3 , lo que evidencia que existe una tendencia asimétrica hacia condiciones cálidas con periodos más intensos, pero de menor duración que las más frías (Fig. 8). Al respecto, vale recordar que este ecosistema de afloramiento está localizado en la zona tropical, lo que le infiere calidez al ecosistema.

En la serie que se analiza, se observa que hacia finales del siglo XX se han producido anomalías positivas destacables (1982 1983 y 1997 - 1998), con una repetición en intervalos de quince años, no observada antes. Esto ha inducido a que algunos autores sugieran que, como consecuencia del calentamiento global, se habrían acelerado las manifestaciones de El Niño y su intensidad hacia finales del siglo pasado. Pero, si se tiene en cuenta lo observado en el SOI, se apreciará que ésta es una manifestación de máxima variabilidad correspondiente a la última cuarta parte del siglo XX. Al respecto, podríamos concluir que las varianzas de las TSM de Chicama presentan patrones de variabilidad que siguen la misma tendencia del SOI y su varianza. De esta manera podríamos dividir la distribución entre 1925 a 1974 y entre 1975 al 2025, haciéndose notar que antes de 1925 se habría dado una fase similar que se inició en 1876 y se extendió durante el primer cuarto del siglo pasado.

Por otro lado, un análisis por periodos decadales de la misma serie evidencia que existe una clara diferenciación entre periodos cálidos y fríos, como se puede observar en la Figura 9.

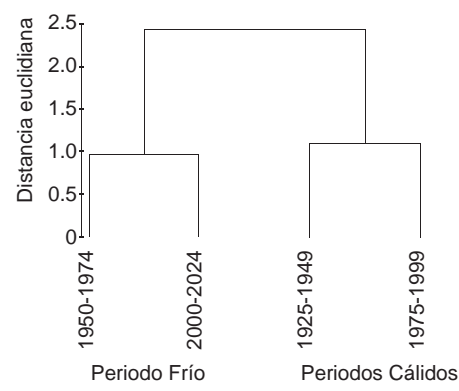

Figura 9. Distancia euclidiana entre periodos fríos y cálidos elaborado a partir de promedios de TSM-Chicama por cuartos de siglo.

Figure 9. Euclidean distance between cold and warm periods from quarter-century averages of Chicama SST.

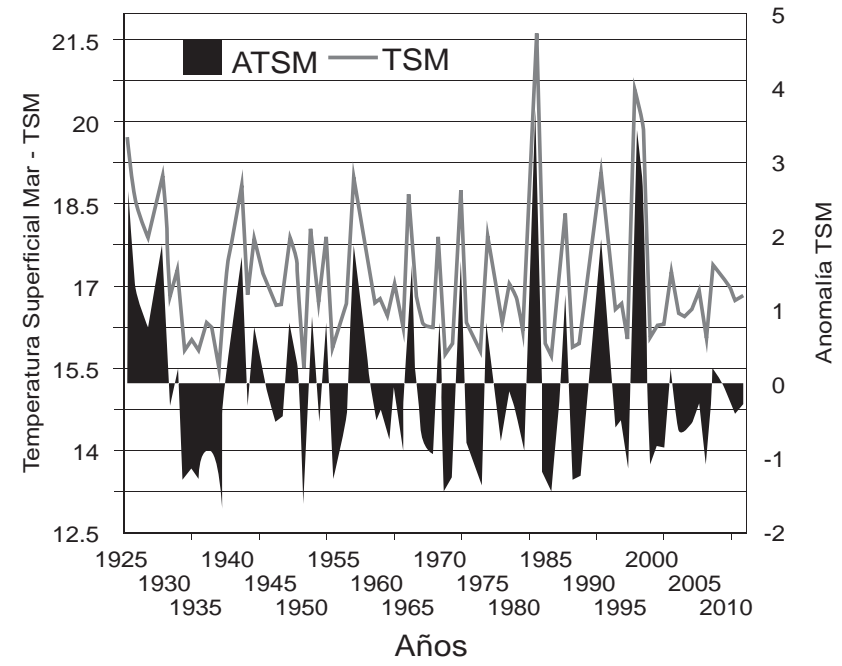

Figura 8. Temperatura Superficial del Mar (TSM) y sus Anomalías (ATSM) en Chicama-Perú, años 1925 - 2012.

Figure 8. Sea Surface Temperature (SST) and its Anomalies (SSTA) in Chicama, Peru, years $1925-2012$.

Áreas de temperatura superficial del mar a partir de imágenes satelitales.- Se obtuvieron promedios anuales entre 1982 y 2012 del área ocupada por las isotermas presentes en el mar peruano y aguas adyacentes, en el espacio delimitado entre los $02^{\circ} \mathrm{N}$ y $20^{\circ} \mathrm{S}$ y entre los 68 y $85^{\circ} \mathrm{W}$, de lo cual se obtuvo patrones de correlación que configuran grupos equivalentes a masas de agua, según se presenta en la Figura 10. Para las temperaturas menores $\left(13\right.$ a $20^{\circ} \mathrm{C}$ ), los coeficientes de correlación (R) estuvieron entre 0.7 y 0.9 que corresponderían a las Aguas Costeras Frías (ACF) (Wyrtki 1967, Zuta \& Guillen 1970, Morón \& Sarmiento 2001). Un segundo grupo con temperaturas entre 20 y $22{ }^{\circ} \mathrm{C}$ presentó correlaciones bajas, entre $-0.25 \mathrm{y}+0.29$, a las que se definió como aguas de mezcla. Las siguientes, entre 22 y $26^{\circ} \mathrm{C}$, resultaron con correlaciones entre 0.7 y 0.9 que serían las Aguas Subtropicales Superficiales (ASS) (Wyrtki 1967, Zuta \& Guillen, 1970, Morón \& Sarmiento 2001). Finalmente, las correspondientes al rango entre 26 y 28 ${ }^{\circ} \mathrm{C}$ presentaron correlaciones entre 0.9 y 1 y se definieron como

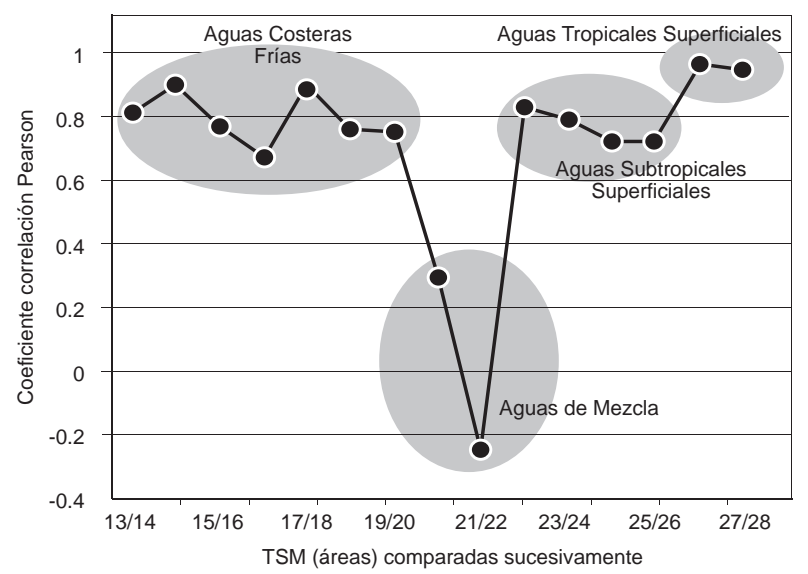

Figura 10. Coeficientes de correlación de Pearson entre las áreas que corresponden a las series de isotermas $\left(13\right.$ a $\left.28^{\circ} \mathrm{C}\right)$ en el mar peruano y aguas adyacentes $\left(02^{\circ} \mathrm{N}-20^{\circ} \mathrm{S}\right.$ y $\left.68^{\circ} \mathrm{W}-85^{\circ} \mathrm{W}\right)$.

Figure 10. Pearson correlation coefficients between the areas corresponding to the series of isotherms $\left(13-28^{\circ} \mathrm{C}\right)$ in the Peruvian sea and adjacent waters $\left(02^{\circ} \mathrm{N}-20^{\circ} \mathrm{S}\right.$ and $\left.68^{\circ} \mathrm{W}-85^{\circ} \mathrm{W}\right)$. 
Aguas Tropicales Superficiales (ATS) (Wyrtki 1967, Morón \& Sarmiento 2001). Entre 1982 y 2012, con estas consideraciones se definen escenarios térmicos que se diferencian claramente a partir del año 2000 (Fig. 11) y si se comparan con la tendencia de la Temperatura Superficial Media Ponderada (TSMP), la tendencia es muy similar a la que corresponde a las áreas de las isotermas comprendidas entre 22 y $25^{\circ} \mathrm{C}$ (ASS).

De esta manera, las condiciones térmicas del mar peruano estarían regidas por la interacción acercamiento-alejamiento de las ASS y las ACF, lo cual define escenarios que constituyen estados ambientales fríos y cálidos. En esa medida, a partir de las áreas de TSM determinadas a través de imágenes satelitales, debemos concluir que durante las décadas de los ochenta y de los noventa han prevalecido condiciones cálidas en el mar peruano y a partir del 2000 se ha iniciado un periodo con dominancia de condiciones frías. Al respecto, subrayamos que en los últimos 12 ańos se ha reducido la TSMP en $1.4^{\circ} \mathrm{C}$ debido a un incremento significativo de las áreas de las isotermas comprendidas entre 13 y $20^{\circ} \mathrm{C}$ correspondiente a las ACF.

Por otro lado, si se relacionan las denominadas ACF y ASS con las variables ambientales de macroescala (Tabla 3) como la TSM-Chicama, TSMP, SOI, PDO y MEI, las correlaciones son significativas, evidenciando que lo obtenido a partir de imágenes satelitales explica parte del comportamiento del ecosistema de esta zona del Pacífico Sudoriental.

La pesquería del jurel y las variables de macroescala en el mar peruano.- El jurel T. murphyi es un recurso pelágico que cuenta con registros históricos de desembarques desde la década de los treinta. Niquen et al. (2013) describen detalladamente esta pesquería, documentando registros de desembarque y otros datos pesqueros desde comienzos del siglo XX. Los primeros ańos muestran valores bajos porque la pesquería dirigida a esta especie era muy reducida; los registros de desembarques recién aumentaron en la segunda mitad de la década de los setenta, llegando a 504992 y 386793 toneladas en 1977 y 1978 respectivamente. En la década de los ochenta, los desembarques fueron más bajos, pero en 1985 llegaron a 189000 toneladas y hacia finales de la década superaban las 100000 toneladas. La década de los noventa mostro un incremento de los desembarques, manteniéndose en promedio por encima de las 200000 toneladas. En el 2001, los desembarques superaron las 700000 toneladas, y después descendieron paulatinamente hasta alcanzar su mínima expresión en el 2010, para luego volver a recuperarse por encima de las 200000 toneladas el 2011. En general, los desembarques no mostraron una tendencia determinada, predominando una alta variabilidad.

Para el caso del jurel T. murphyi en el mar peruano, por la elevada variabilidad que condiciona un alto desplazamiento del recurso, las biomasas acústicas sólo expresan su disponibilidad y muestran dos momentos claramente diferenciados (Fig. 12). El primero corresponde a las décadas de los ochenta y los noventa, que coinciden con ATSMP positivas y altas biomasas, las cuales promedian los 4.9 millones de toneladas; y el segundo, que corresponde a los años 2000 con ATSMP negativas y con bajas biomasas que en promedio no superan las 500000 toneladas anuales. Es decir, en los últimos años la biomasa ha mostrado una reducción del 90\%. Sin embargo, las capturas han mostrado un incremento promedio del $30 \%$, lo que se explica en que, si bien las biomasas son mayores durante la dominancia de ATSMP

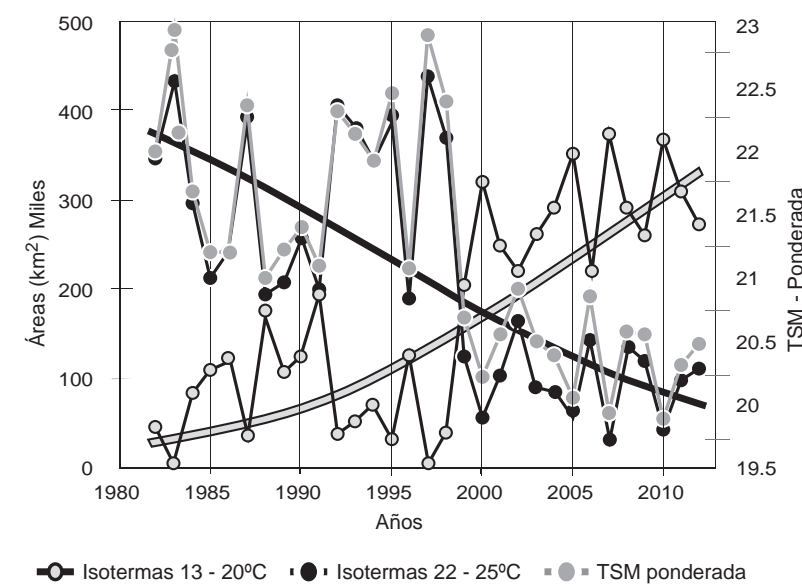

Figura 11. Distribución de áreas de conjunto de isotermas $\left(13-20^{\circ} \mathrm{C}\right.$ y $\left.22-25^{\circ} \mathrm{C}\right)$ en el mar peruano y áreas adyacentes $\left(02^{\circ} \mathrm{N}-20^{\circ} \mathrm{S}\right.$ y 68 $-85^{\circ} \mathrm{W}$ ), curvas de tendencia de las ASS (líneas negra) y ACF (línea gris), y Temperatura Superficial Media del Mar Ponderada (TSMP).

Figure 11. Distribution of the areas two sets of isotherms $\left(13-20^{\circ} \mathrm{C}\right.$ and $\left.22-25^{\circ} \mathrm{C}\right)$ in the Peruvian sea and adjacent waters $\left(02^{\circ} \mathrm{N}-20^{\circ} \mathrm{S}\right.$ and $68-85^{\circ} \mathrm{W}$ ), trend curves of the SSW (black line) and CCW (grey line), and Weighted Average Sea Surface Temperature (WASST).

positivas, su distribución en el mar peruano es más amplia, siendo menos accesible a la flota pesquera de cerco dedicada a la pesca de este recurso. Asimismo, durante los periodos cálidos, las mínimas de oxígeno (Bertrand et al. 2011) y la isoterma de 15 ${ }^{\circ} \mathrm{C}$ (Flores et al. 2013) se ubican a mayor profundidad, lo cual favorece la dispersión vertical del jurel T. murphyi, haciendolo menos vulnerable a la pesquería de cerco. A esto se debe agregar que durante los últimos 50 ańos las principales especies objetivo de la pesquería peruana de cerco han sido la anchoveta $E$. ringens en la década de los sesenta, la sardina $S$. sagax sagax de mediados de la década de los setenta hasta inicios de los noventa, y luego nuevamente la anchoveta, persistiendo hasta la actualidad (2013).

En general, hasta hace algunos años la flota peruana se ha dedicado a la pesca de jurel T. murphyi más como una alternativa para los periodos en que las otras pesquerías eran poco abundantes o no se ejercían.

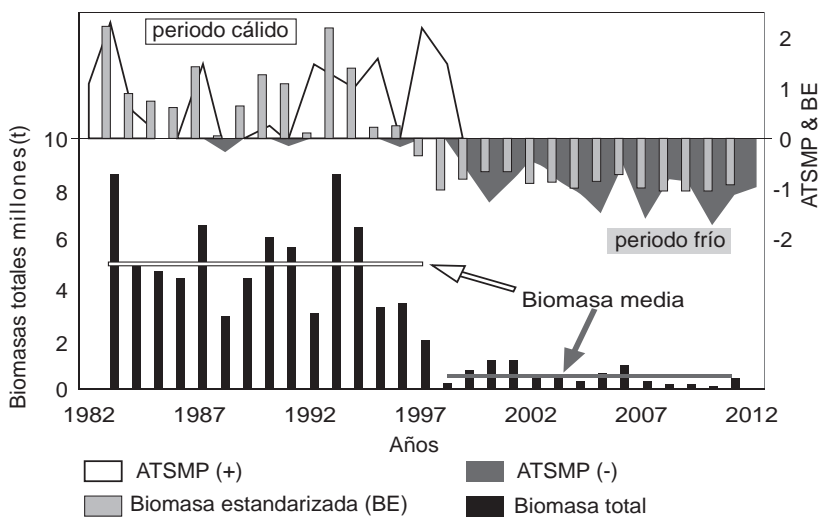

Figura 12. Biomasas acústicas totales y biomasas estandarizadas de jurel T. murphyi y ATSMP, evidenciando periodos cálidos con dominancia de ASS y desviaciones positivas de las biomasas, y periodos fríos con predominio de ACF y desviaciones negativas de las biomasas.

Figure 12. Total acoustic biomass and standardized biomass of Jack mackerel T. murphyi, and Weighted Surfase See Temperature Anomaly (WSSTA) showing warm periods with dominance of SSW and positive deviations of biomass and cold periods with CCW prevalence and negative biomass deviations. 
Por otro lado, si se analizan los desembarques (capturas) y las biomasas acústicas de jurel T. murphyi (Tabla 3) en relación a variables de macroescala se observa que las primeras muestran una baja relación con las variables ambientales, debido a que las capturas estarían dependiendo más de decisiones de orden económico-sociales y de manejo pesquero que de la real disponibilidad y abundancia del recurso. Sin embargo, para el caso de las biomasas acústicas es notable su relación positiva con las ASS, TSM-Chicama, TSMP, PDO y MEI y su relación negativa con el SOI, dado que las manifestaciones negativas de este último explican anomalías positivas en el mar peruano y zona ecuatorial. Asimismo, se destaca la relación negativa con las ACF, lo que resulta natural en un recurso cuyo patrón de abundancia es mayor cuando predominan condiciones cálidas en el ecosistema de afloramiento peruano.

El análisis de similitud (similaridad) de Bray-Curtis de las biomasas acústicas de jurel T. murphyi, de 1983 a 2011, evidencia agrupamientos claramente diferenciados entre las décadas de los ochenta y los noventa (periodo cálido) y las décadas de los 2000 (periodo frío), lo que se verifica también en un análisis de agrupamiento utilizando distancia euclidiana (Figs. 13A y 13B). $\mathrm{Al}$ respecto, las biomasas de jurel T. murphyi correspondientes al stock norte (far north) que se distribuye frente a las costas de Ecuador, Perú, y posiblemente en el norte de Chile, dependerian del acercamiento y extensión de las isotermas entre 22 y $25^{\circ} \mathrm{C}$ (ASS), cuya posición y dimensión están asociadas a variables de macroescala que gobiernan los ciclos interdecadales en el océano Pacífico. En esa medida, los cambios trascendentales de las biomasas de jurel T. murphyi del orden de 10 a 1 y viceversa sólo explican cambios en la disponibilidad del recurso frente a condiciones favorables o adversas, según sea el caso, pero no necesariamente una reducción de la biomasa en la misma magnitud.

Lo extraído por la pesquería peruana no explica tal reducción, pues las capturas sólo han representado el 5\% de la biomasa estimada como promedio entre 1983 y 1997 . Dicha cifra resulta representativa para todo el stock norte (far north), puesto que en el mismo periodo los desembarques de jurel $T$ murphyi fueron muy reducidos en el Ecuador y en el norte de Chile.

\section{La pesquería del jurel y otros recursos y sus pesquerías}

El jurel T. murphyi no es la única especie que se presenta en abundancia en el mar peruano y cuyos cambios en abundan-
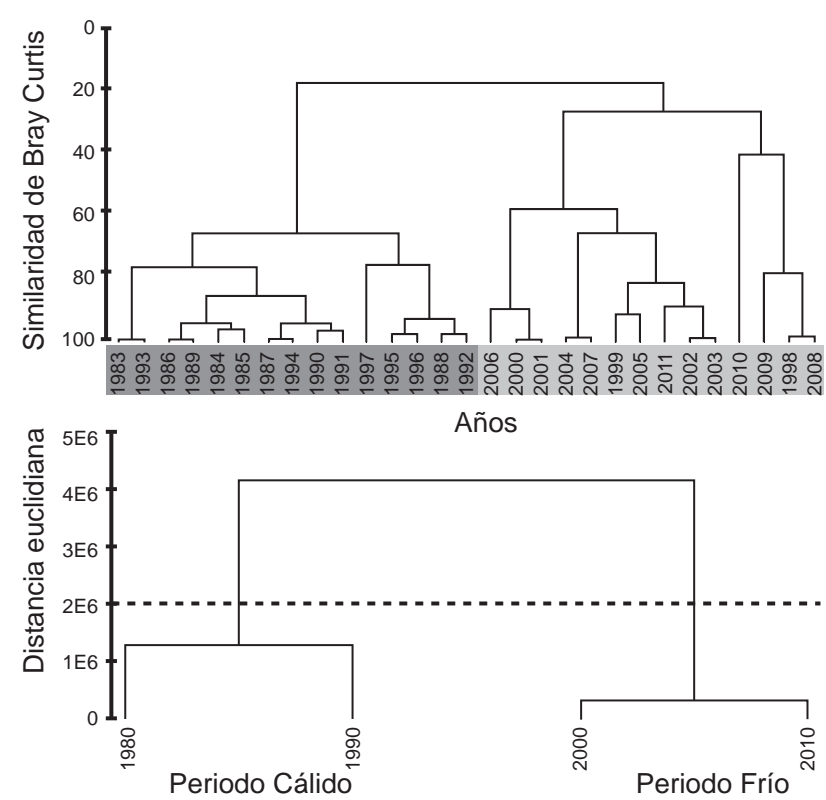

Figura 13. (A) Resultados del análisis de agrupamiento por similitud de Bray Curtis de biomasas acústicas de jurel T. murphyi de 1983 a 2011. (B) Agrupamiento por distancia euclidiana de las biomasas de jurel T. murphyi de 1983 al 2011.

Figure 13. (A) Results of Bray Curtis similarity grouping analysis of acoustic biomass of Jack mackerel T. murphyi 1983 - 2011. (B) Grouping by Euclidean distance of Jack mackerel T. murphyi biomass from 1983 to 2011.

cia y disponibilidad están influenciados por las condiciones ambientales y su interacción con otras especies, con las cuales comparte el ecosistema.

$\mathrm{Al}$ respecto, el análisis de las interacciones del recurso jurel $T$. murphyi en el mar peruano revela que existe una fuerte relación con la caballa $S$. japonicus y la merluza $M$. gayi peruanus, al parecer especies asociadas al mismo sistema de circulación representado por las ASS (Aguas Subtropicales Superficiales) y la ESCC (Extensión Sur de la Corriente de Cromwell) (Figs.14A y 14B).

Este análisis concuerda con la observación de Niquen et al. (2013) respecto a la coincidencia en los picos de capturas en los ańos 1977 y 1978 para jurel T. murphyi y merluza M. gayi peruanus en el Perú, y entre 1994 y 1998 y en 2001 para jurel T. murphyi en Ecuador y Perú así como para merluza M. gayi

Tabla 3. Relación entre los promedios decadales de biomasa, captura y variables ambientales de macroescala. Aguas Costeras Frías (ACF), Aguas Subtropicales Superficiales (ASS), Temperatura Superficial del Mar-Chicama (TSM-Chi), Temperatura Superficial del Mar Ponderada (TSMP), Índice Oscilación del Sur (SOI), Oscilación Decadal del Pacífico (PDO) e Índice Multivariado ENSO (MEI). Años 1983 - 2011 promediados por décadas $(1980-1990-2000-2010)$

Table 3. Relationship between decadal averages of biomass (biomasa), catch (captura) and macro-scale environmental variables. Cold Coastal Waters (CCW/ACF), Subtropical Surface Waters (SSW/ASS), Sea Surface Temperature-Chicama (SST-Chi/TSM-Chi) Weighted Sea Surface Temperature (WSST/TSMP), Southern Oscillation Index (SOI/IOS), Pacific Decadal Oscillation (PDO), Multivariate ENSO Index (MEI). Years $1983-2011$ averaged by decades $(1980-1990-2000-2010)$

\begin{tabular}{cccccccccc}
\hline & Biomasa & Captura & ACF & ASS & TSM-Chi & TSMP & IOS & PDO & MEI \\
\hline Biomasa & 1.000 & -0.228 & -0.969 & 0.952 & 0.698 & 0.961 & -0.687 & 0.868 & 0.759 \\
Captura & -0.228 & 1.000 & -0.019 & 0.047 & 0.279 & 0.029 & -0.479 & -0.531 & 0.421 \\
ACF & -0.969 & -0.019 & 1.000 & -0.994 & -0.806 & -0.997 & 0.816 & -0.744 & -0.877 \\
ASS & 0.952 & 0.047 & -0.994 & 1.000 & 0.866 & 0.999 & -0.786 & 0.681 & 0.855 \\
TSM-Chi & 0.698 & 0.279 & -0.806 & 0.866 & 1.000 & 0.845 & -0.596 & 0.251 & 0.677 \\
TSMP & 0.961 & 0.029 & -0.997 & 0.999 & 0.845 & 1.000 & -0.793 & 0.709 & 0.861 \\
IOS & -0.687 & -0.479 & 0.816 & -0.786 & -0.596 & -0.793 & 1.000 & -0.488 & -0.992 \\
PDO & 0.868 & -0.531 & -0.744 & 0.681 & 0.251 & 0.709 & -0.488 & 1.000 & 0.532 \\
MEI & 0.759 & 0.421 & -0.877 & 0.855 & 0.677 & 0.861 & -0.992 & 0.532 & 1.000 \\
\hline
\end{tabular}



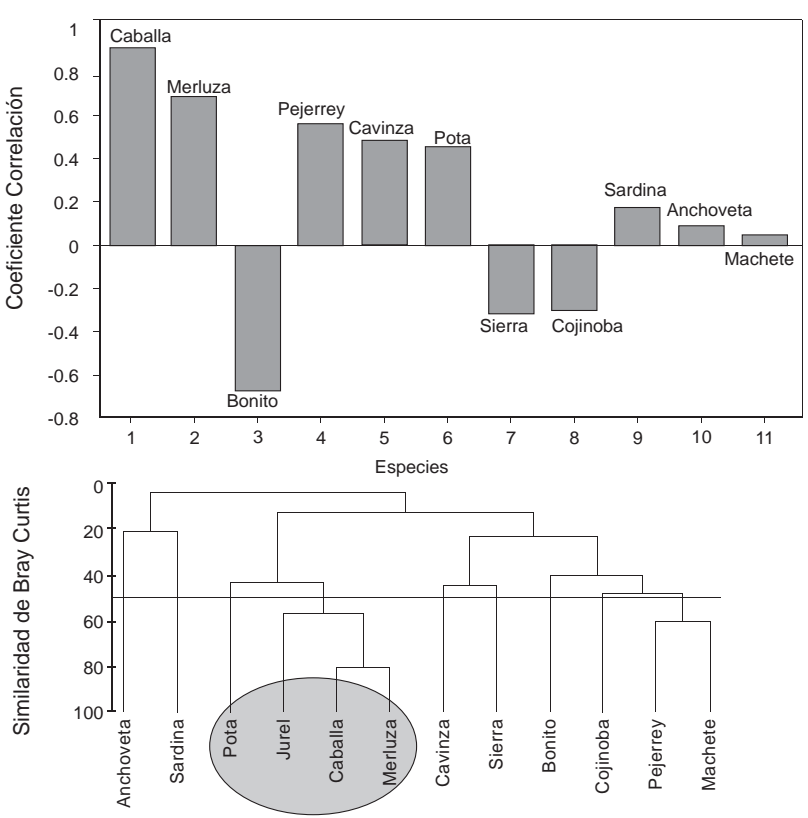

Figura 14. (A) Correlación de Pearson entre los desembarques de jurel T. murphyiy y los de otras especies seleccionadas (1960-2012). (B) Agrupamiento por similitud de Bray-Curtis de desembarques (1960 - 2012) de las principales especies del ecosistema de afloramiento peruano.

Figure 14. (A) Pearson's correlation between the landings of Jack mackerel T. murphyi and that of other selected species (1960-2012). (B) Results of Bray Curtis similarity grouping analysis of landings (1960 -2012 ) of the main species of the Peruvian upwelling ecosystem.

peruanus en el Perú, coincidentes con desviaciones positivas del PDO. Esto sugiere la posibilidad de pronosticar periodos favorables para la pesca de jurel T. murphyi y merluza $M$. gayi peruanus en Perú y Ecuador a partir de expresiones positivas del PDO. Sin embargo, las desviaciones positivas del PDO estarán seguidas de desviaciones negativas, y viceversa, por el periodo de alta variabilidad ambiental por el que atravesamos. En ese sentido, dado que en los últimos años han predominado condiciones frías (en 4 años de 5), es de esperar que en los próximos años se mantenga la predominancia de manifestaciones positivas del PDO, lo cual favorecería la pesquería de jurel T. murphyi para Ecuador, Perú y el norte de Chile, según se ha venido dando en los últimos años.

Por otro lado, son menos significantes las relaciones con pejerrey $O$. regia regia, cabinza $I$. conceptionis y pota $D$. gigas. Los desembarques de esta última especie han mostrado un incremento, en contraste con lo acaecido con jurel T. murphyi en los últimos años, lo que explica la diferencia con lo obtenido por Espino y Yamashiro (2012), con información hasta el 2009.

Asimismo, es notable la relación inversa con bonito $S$. chiliensis chiliensis, seguido de sierra $S$. sierra y cojinova $S$. violacea, pero en mucha menor magnitud. Un análisis de similitud de Bray-Curtis entre las principales especies de la pesquería peruana revela una asociación entre jurel T. murphyi, caballa $S$. japonicus, merluza $M$. gayi peruanus y, en menor grado, con la pota $D$. gigas, lo cual indicaría tendencias similares en su disponibilidad a las pesquerías. La asociación jurel-caballa se explica por la relación con las ASS, y la asociación jurel-merluza por la relación con la ESCC. En tanto que en la relación jurel-pota, debido a que los frentes entre ASS y ACF son más amplios en periodos de abundancia de jurel T. murphyi, el desarrollo de la pota $D$. gigas se posibilita (Arguelles \& Tafur 2010, Niquen et al. 2004, Swartzman et al. 2008).

\section{Discusión}

El Sistema de la Corriente Peruana está sometido a patrones de variabilidad decadales y multidecadales que afectan la dinámica de circulación oceánica y los patrones de distribución, abundancia y disponibilidad de los recursos pesqueros, en la medida que las principales masas de aguas ACF y ASS alternan su dominancia.

En esa medida, se encuentran evidencias de que las variaciones seculares estarían vinculadas a fluctuaciones de amplia escala del SOI, como se reflejan en oscilaciones detectadas para el Pacífico Norte en anillos de árboles de Baja California, lo cual sugiere la existencia de éstas en el clima de esta región entre 1706 y 1977 , con grandes oscilaciones decadales en 1750, 1905 y 1947 (Biondi et al. 2001), con una intermitencia de casi 50 años en el último siglo. Asimismo, en un rango de escalas de tiempo que se remontan por lo menos a 1600 ańos, reconstrucciones climáticas a partir de anillos de árboles y de corales del Pacífico sugieren variaciones de PDO, existiendo variaciones importantes en diferentes periodos. Sin embargo, para el siglo XX las fluctuaciones PDO fueron más enérgicas con dos periodicidades generales, una de 15 a 25 años, y otra de 50 a 70 años (Mantua \& Hare 2002).

En otras latitudes, también existen evidencias del efecto de los regímenes ambientales en los recursos pesqueros. Como ejemplos más saltantes, están los casos de la influencia de regímenes ambientales en la dinámica poblacional del lenguado del Pacífico Norte "halibut" Hippoglossus stenolepis, el colín de Alaska o "Alaska pollock", Theragra chalcogramma, la sardina de California Sardinops caeruleus (=Sardinops sagax), la sardina japonesa $S$. melanostictus (=Sardinops sagax) y los lenguados del mar de Bering: Hippoglossoides elassodon, Lepidopsetta polyxystra y Atheresthes stomias, entre los más importantes, y en cuyos casos se han documentado efectos sobre los reclutamientos, procesos de denso-dependencia y patrones de crecimiento individual y poblacionales (Quinn \& Niebauer 1995, Jacobson \& MacCall 1995, Wada \& Jacobson 1998, Francis et al. 1998, Walters \& Wilderbuer 2000, Clark \& Hare 2002).

Con mayor frecuencia, los elementos determinantes que explican la variabilidad ambiental en el Pacífico Norte son asociados al PDO (Hare \& Mantua 2001, Lluch-Cota et al. 2003) y en el hemisferio sur al fenómeno ENSO (Mantua et al. 1997). Aunque en el hemisferio sur se hace principalmente referencia al fenómeno ENSO, existe la posibilidad de que se produzcan procesos de oscilación interdecadales que pueden influenciar la dinámica poblacional de los recursos en formas y a niveles diferentes. Estos procesos han sido descritos por Chávez et al. (2003), quienes plantean la existencia de ciclos ambientales de alrededor de 50 años, en los que se alternan fases frías y cálidas. Las fases frías en los periodos de 1900 - 1925 y de 1950 - 1975 y las fases cálidas en los periodos de 1925 - 1950 y de 1975 a finales del siglo pasado indican que en el momento actual estamos atravesando por una fase fría con dominancia de anchoveta $E$. ringens y ausencia de sardina $S$. sagax y, asimismo, por el alejamiento y disminución ostensible de la disponibilidad de jurel T. murphyi.

Por otro lado, la variabilidad decadal también puede modular la frecuencia, intensidad y duración del ciclo ENSO (Wang \& 
Picaut 2004, Wang et al. 2004, Wang \& Fiedler 2006, Cobb et al. 2003) así como el momento de inicio de El Niño (Espino 2003). Estos cambios de baja frecuencia en el entorno físico pueden resultar en "cambios de régimen" en la estructura del ecosistema marino en la cuenca del Pacífico (Chávez et al. 2003, Mantua \& Hare 2002, Schwartzlose et al. 1999, Hsieh et al. 2005, Di Lorenzo \& Ohman 2013).

Dentro del Ecosistema de la Corriente Costera Peruana se identifican dos "regímenes de anchoveta E. ringens" (desde el inicio de la pesca en la década de los cincuenta hasta inicios de la década de los setenta, y desde principios de la década de los noventa hasta la actualidad), separados por un "régimen de sardina S. sagax" (a partir de mediados de la década de los setenta hasta principios de la década de los noventa) (Csirke at al. 1996, Chávez et al. 2003, Alheit \& Niquen 2004, Ayón et al. 2011).

Por otro lado, Ballón et al. (2008) describen cambios en las tallas de merluza $M$. gayi peruanus, diferenciándolas entre las décadas de los setenta y ochenta con respecto a la de los noventa y la década del 2000 al 2010, de un rango de 35 a $50 \mathrm{~cm}$ a otro de 20 a $35 \mathrm{~cm}$, respectivamente, lo cual evidencia una maduración más temprana y desaparición de adultos por presión de la pesca (Wosnitza-Mendo et al. 2004, Guevara-Carrasco 2004, Guevara-Carrasco \& Lleonart 2008).

Sin embargo, anteriormente se ha definido (Niquen et al. 2013) una relación entre jurel T. murphyi y merluza M. gayi peruanus, basándose en que ambas especies están asociadas a una dominancia de las ASS que favorece la extensión de la ESCC. Por lo tanto, tal reducción de tallas estaría también asociada a cambios en los patrones de distribución por tallas del recurso, que se afectan por la retracción de la ESCC, lo que explicaría la presencia de importantes biomasas de merluza $M$. gayi peruanus en el mar ecuatoriano (328000 toneladas) el 2010 (González Troncoso 2010).

En el Perú, las merluzas M. gayi peruanus de mayor tamaño se distribuyen hacia el norte y las más pequeńas hacia el sur (Armstrong, 1981; Samamé, 1981; Espino et al. 1995). Con la retracción de ESCC las más pequeñas se ubican en la principal área de pesca de merluza M. gayi peruanus del Perú $(04-05$ oS) y las más grandes en el Ecuador, e incluso más al norte.

Al respecto, se subraya que en la Figura 15, en el caso del jurel T. murphyi y la merluza $M$. gayi peruanus, se aprecia que ambas curvas muestran la misma tendencia, con una relativa estabilidad hasta 1990, excepto en 1987 en que la talla media del primero disminuye drásticamente por debajo de $25 \mathrm{~cm}$ y se recupera hacia 1990. A partir de este año muestra una tendencia declinante que persiste los veinte años siguientes, hasta 2011, con un aumento notorio en 2012. Al estar sometidas al mismo régimen de circulación oceánica y a los mismos patrones de variabilidad de macroescala, ambas especies siguen el comportamiento de las condiciones que les son propias. La merluza $M$. gayi peruanus está vinculada a la ESCC que al retraerse hacia el norte retrae consigo a los individuos de mayor tamaño, dejando a disponibilidad de la pesquería los más pequeños, cuya ubicación era más al sur en la década de los setenta y ochenta.

Por su parte, el jurel T. murphyi se aleja en la medida que las ASS se alejan, portando entre sí a los individuos de mayor tamaño y dejando disponibles los más pequeños en la zona costera.

Gutierrez et al. (2008) caracterizan este periodo (mediados de los noventa al año 2000) como de condiciones más frías, debido al dominio de aguas frías costeras a lo largo del Perú, basándose en las relaciones existentes entre munida Pleuroncodes monodon y anchoveta $E$. ringens. Esta característica favorece el incremento de la productividad primaria y la producción de zooplancton (Swartzman et al. 2008, Ayón et al. 2008), lo cual diferencia este periodo de uno anterior menos frío o más cálido.

Por su parte Barange et al. (2009) indican que en general las poblaciones de anchoveta $E$. ringens y sardina $S$. sagax expanden sus áreas de distribución con aumentos en su densidad y concentración, en consistencia con el modelo de la cuenca que habitan. La relación entre el área de distribución y biomasa de la población es alométrica, lo que implica la existencia de un límite a la expansión en función de las nuevas áreas. Los patrones de ocupación de los espacios difieren entre la sardina $S$. sagax y la anchoveta $E$. ringens en algunas regiones. En el sur de África y Japón, la anchoveta ocupa un área mayor por unidad de biomasa que la sardina, de acuerdo con los requerimientos de hábitat determinados por su ecología de alimentación. En California y Perú, los resultados confirman que en general, estas especies amplían sus áreas de distribución con el tamaño de la población, pero los modelos específicos son menos claros. Se puede inferir que las poblaciones se acercarán o alejarán de las áreas de pesca en la medida que las condiciones les sean favorables, dando imágenes sesgadas de cambios en la abundancia. Estas imágenes serían más bien reflejos de cambios en la disponibilidad, en función a los cambios en las condiciones del ambiente (Vert- pre et al. 2013), pero no necesariamente cambios en la abundancia o biomasa total.

Finalmente, se reconocen patrones decadales con cambio de régimen o salto climático alrededor de 1925/1926 y 1976/1977 hacia un periodo cálido, con manifestaciones en el ambiente y en el comportamiento de recursos pesqueros (Noakes et al. 2000, McFarlane \& Beamish 2001, Wilderbuer 2002, Lavaniegos \& Ohman 2003, Peterson \& Schwing 2003, Gray et al. 2003, Miller \& Sydeman 2004, Hoff 2006, Saitoh 2006, Chhak \& Di Lorenzo 2007, d'Orgeville \& Peltier 2007, Zhuguo Ma 2007, Noakes \& Beamish 2009, Jacques-Coper 2009, Ceballos et al. 2009).

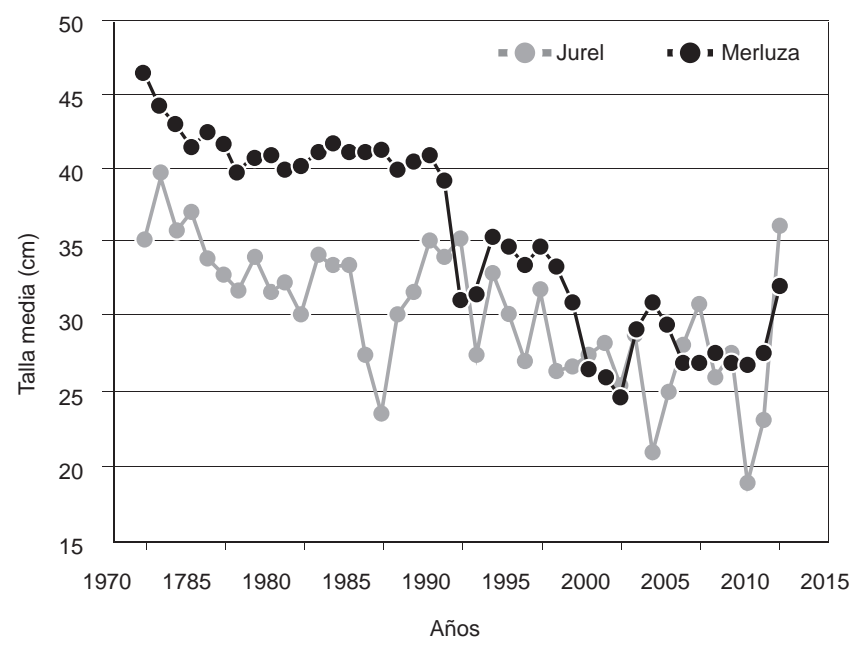

Figura 15. Tallas medias anuales de jurel T. murphyi (gris) y merluza M. gayi peruanus (negro) en las capturas comerciales, Perú, 1972 a 2012

Figure 15. Annual average sizes of Jack mackerel T. murphyl (grey) and hake M. gayi peruanus (black) in commercial catches, Peru, $1972-2012$ 
En conclusión, los cambios en magnitud detectados en las biomasas observadas de jurel T. murphyi en el mar peruano están reflejando cambios en la disponibilidad del recurso asociados a patrones de variabilidad de macroescala seculares y multidecadales. A su vez, estos patrones afectan también las manifestaciones de El Niño haciendo que sean otońales o primaverales si el PDO imperante es negativo o positivo, respectivamente (Espino 2003). Asimismo, las fluctuaciones en la disponibilidad del recurso están afectando las pesquerías de Ecuador, Perú y norte de Chile, las mismas que han mostrado variaciones importantes en sus desembarques y habrían dado una imagen sesgada de su abundancia, lo que conduce a diagnósticos errados de la real situación del recurso. Por ello, es necesario que se promuevan programas de investigación mancomunados más activos entre los países costeros de la región que participan en la pesca del stock norte (far north) de jurel T. murphyi, con el objeto de conocer su real dimensión y dinámica, en función de las variables ambientales que gobiernan la parte norte del Ecosistema de la Corriente Costera Peruana.

\section{Agradecimientos}

Deseo expresar mi agradecimiento al ingeniero Luis Escudero por haber proporcionado las áreas satelitales de las diferentes isotermas $\left(13-28^{\circ} \mathrm{C}\right)$ en la serie $1982-2011$. A Carmen Yamashiro por la revisión del trabajo y sus valiosas sugerencias para mejorarlo.

\section{Literatura citada}

Armstrong, D. 1981. Investigación de la merluza en IMARPE. Informe Inst. Mar. Perú, Callao, 79 I parte. $47 \mathrm{p}$.

Alheit J. \& M. Niquen. 2004. Regime shifts in the Humboldt Current ecosystem. Progress in Oceanography): $201-222$. DOI: $10.1016 / \mathrm{j}$. pocean.2004.02.006

Argüelles J. \& R. Tafur. 2010. New insights on the biology of the jumbo squid Dosidicus gigas in the Northern Humboldt Current System: Size at maturity, somatic and reproductive investment. Fisheries Research 106: 185 - 192. Doi 10.1016/j.fishres.2010.06.005

Ayón P., G. Swartzman, P. Espinoza \& A. Bertrand. 2011. Longterm changes in zooplankton size distribution in the Peruvian Humboldt Current System: conditions favouring sardine or anchovy. Mar Ecol. Prog. Ser. 422: 211 - 222. DOI: 10.3354/meps08918

Ayón P., G. Swartzman, A. Bertrand, M. Gutiérrez \& S. Bertrand. 2008. Zooplankton and forage fish species off Peru: Large - scale bottom - up forcing and local - scale depletion. Progress in Oceanography 79: $208-214$.

Ballón M., C. Wosnitza-Mendo, R. Guevara-Carrasco \& A. Bertrand. 2008. The impact of overfishing and El Niño on the condition factor and reproductive success of Peruvian hake, Merluccius gayi peruanus. Progress in Oceanography 79: 300 - 307. DOI: 10.1016/j.pocean.2008.10.016

Barange M., J. Coetzee, A. Takasuka, K. Hill, M. Gutiérrez, Y. Oozeki, C. van der Lingen \& V. Agostini. 2009. Habitat expansion and contraction in anchovy and sardine populations. Progress in Oceanography 83(1-4): $251-260$.

Bertrand A., A. Chaigneau, S. Peraltilla, J. Ledesma, M. Graco, F. Monetti \& Francisco P. Chávez. 2011. Oxygen: A Fundamental Property Regulating Pelagic Ecosystem Structure in the Coastal Southeastern Tropical Pacific. PLoS ONE 6(12): e29558. doi:10.1371/journal. pone.0029558.

Biondi F., A. Gershunov \& D.R. Cayan, 2001: North Pacific Decadal Climate Variability since 1661. Journal of Climate, 14: 5 - 10. doi: http:// dx.doi.org/10.1175/1520-0442(2001)014<0005:NPDCVS >2. $0 . \mathrm{CO} ; 2$

Ceballos L., E. Di Lorenzo, N. Schneider \& B. Taguchi. 2009. North Pacific Gyre Oscillation synchronizes climate fluctuations in the eastern and western North Pacific. Journal of Climate, DOI: 10.1175/2009JCLI2848.1.

Chhak K.\& E. Di Lorenzo. 2007. Decadal variations in the California Current upwelling cells, Geophys. Res. Lett. 34, L14604, doi:10.1029/2007GL030203.
Chávez F.P., J. Ryan, S.E. Lluch-Cota \& M. Niquen. 2003. From anchovies to sardines and back: Multidecadal change in the Pacific Ocean. Review: Climate. Science 299: 217 - 221. DOI: 10.1126/science. 1075880

Clark W.G. \& S.R. Hare. 2002. Effects of Climate and Stock Size on Recruitment and Growth of Pacific Halibut. N. Am. J. Fish. Mgmt. 22: $852-862$.

Cobb K.M., C.D. Charles, H. Cheng \& R.L. Edwards. 2003. El Niño Southern Oscillation and tropical Pacific climate during the last millennium. Nature 424: 271 - 276. doi:10.1038/nature01779

Csirke J., R. Guevara - Carrasco, G. Cárdenas, M. Niquen \& A. Chipollini. 1996. Situación de los recursos anchoveta (Engraulis ringens) y sardina (Sardinops sagax sagax) a principios de 1994 y perspectivas para la pesca en el Perú de 1994, conparticular referencia a las regiones norte y centro de la costa peruana. Bol. Inst. Mar Perú Callao 15(1): $1-23$.

Di Lorenzo E. \& M. D. Ohman. 2013. A double - integration hypothesis to explain ocean ecosystem response to climate forcing. PNAS 110 (7): 2496-2499. DOI: 10.1073/pnas.1218022110

d'Orgeville M. \& W.R. Peltier. 2007. On the Pacific Decadal Oscillation and the Atlantic Multidecadal Oscillation: Might they be related? Geophys. Res. Lett. 34, L23705, doi:10.1029/2007GL031584.

Espino, M., R. Castillo \& F. Fernández. 1995. Biology and Fisheries of Peruvian Hake (M. gayi peruanus). P. 339 - 359. In J. Alheit and T. Pitcher (eds). HAKE, fisheries, ecology and markets. Chapman \& Hall, Fish and fisheries Series 15. First edition.

Espino M. 2003. Estrategia de gestión oriental para el Pacífico Oriental con especial mención a la pesquería peruana. Tesis para optar el grado de Magíster en Geografía con mención en Ordenamiento y Gestión Ambiental. Unidad de Post Grado, Facultad de Ingeniería Geológica, Minera, Metalúrgica y Geográfica, Universidad Nacional Mayor de San Marcos, Lima. 102 pp.

Espino M. \& C. Yamashiro. 2012. La variabilidad climática y las pesquerías en el Pacífico Sudoriental. En: P.M. Arana (Guest Editor). International Conference: "Environment and Resources of the South Pacific". Latin American Journal of Aquatic Research 40(3): 705 - 721. DOI: $103856 /$ vol 40 - issue3 - fulltext - 18.

Flores R., M. Espino, G. Luque \& J. Quispe. 2013. Patrones de variabilidad ambiental en el mar peruano. En: Csirke J., R. Guevara-Carrasco \& M. Espino (Eds.). Ecología, pesquería y conservación del jurel (Trachurus murphyi) en el Perú. Rev. peru. biol. número especial 20(1): $021-028$

Francis R.C., S.R. Hare, A.B. Hollowed \& W.S. Wooster. 1998. Effects of interdecadal climate variability on the oceanic ecosystems of the Northeast Pacific Ocean. Fish. Oceanogr. 7: 1 - 21.

González Troncoso D.M. 2010. Informe de la campaña de investigación pesquera. Ecuador 2010. B/O Miguel oliver, 16/10/2010 -05/11/2010. Campańas Oceanográficas de Coorperación Espańa - Ecuador 2008 - 2010. Gobierno de Espańa, Ministerio del Medio Ambiente, Medio Rural y Marino, Instituto Espańol de Oceanografía. República del Ecuador, Instituto Nacional de Pesca. 130 pp.

Gray S.T., J.L. Betancourt, C.L. Fastie \& S.T. Jackson. 2003. Patterns and sources of multidecadal oscillations in drought - sensitive tree - ring records from the central and southern Rocky Mountains. Geophys. Res. Lett. 30(6), 1316, doi:10.1029/2002GL016154.

Guevara-Carrasco R. 2004. Sobrepesca de la merluza peruana: lecciones mal entendidas. Boletín 21(1-2):27-32

Guevara-Carrasco R., \& J. Lleonart. 2008. Dynamics and fishery of the Peruvian hake: Between nature and man. Journal of Marine Systems 71 (3-4): 249-259. doi:10.1016/j.jmarsys.2007.02.030.

Gutiérrez M., A. Ramirez, S. Bertrand, O. Móron \& A. Bertrand. 2008. Ecological niches and areas of overlap of the squat lobster 'munida' (Pleuroncodes monodon) and anchoveta (Engraulis ringens) off Peru. Progress in Oceanography 79: 256-263.

Hare, S.R. 1996. Low frequency climate variability and salmon production. Ph.D. Dissertation. Univ. Washington, School of Fisheries. 306 pp.

Hare S.R. \& N.J. Mantua. 2001. An historical narrative on the Pacific Decadal Oscillation, interdecadal climate variability and ecosystem impacts. Report of a talk presented at the 20th NE Pacific Pink and Chum workshop, Seattle, WA, 22 March 2001.

Hare S.R. \& N.J. Mantua. 2002. The Pacific Decadal Oscillation. Journal of Oceanography 1: 35 - 44, DOI: 10.1023/A: 1015820616384.

Hoff G.R. 2006. Biodiversity as an index of regime shift in the eastern Bering Sea Fish. Bull. 104:226 - 237. 
Hsieh C.H., S.M. Glaser, A.J. Lucas \& G. Sugihara. 2005. Distinguishing random environmental fluctuations from ecological catastrophes for the North Pacific Ocean, Nature 435:336 - 340, doi:10.1038/ nature 02553.

Jacobson L.D. \& A.D. MacCall. 1995. Stock - recruitment models for pacific sardine (Sardinops sagax). Canadian Journal of Fisheries and Aquatic Sciences 52: $566-577$.

Jacques-Coper R. 2009. Caracterización del salto climático de mediados de los setenta en Sudamérica. Primer Congreso de Oceanografía Física, Meteorología y Clima. Universidad de Concepción, Concepción, Chile. 30 septiembre -02 de octubre de 2009. Sesión: Variabilidad Climática y Cambio Global / Paleoclima.

Lavaniegos B.E. \& M.D. Ohman. 2003. Long - term changes in pelagic tunicates of the California Current. Deep - Sea Research II, 50: $2473-2498$.

Lluch-Cota D.B., W.S. Wooster, S.R. Hare, D. Lluch - Belda \& A. Parés - Sierra. 2003. Principal Modes and Related Frequencies of Sea Surface Temperature Variability in the Pacific Coast of North America. Journal of Oceanography 59 (4): 477 - 488 (12). DOI: 10.1023/A: 1025592616562

Maenza R. A. \& R. H. Compagnucci. 2010. Simulación de la pequeña edad de hielo usando el modelo EdGCM. GEOACTA 35: 78 - 91.

Mantua N.J., S.R. Hare, Y. Zhang, J.M. Wallace \& R.C. Francis. 1997. A Pacific interdecadal climate oscillation with impacts on salmon production. Bull. Amer. Meteor. Soc. 78: 1069 - 1079. DOI: http://dx.doi. org/10.1175/1520-0477(1997)078<1069:APICOW>2.0.CO;2

Mantua, N.J. 1999 (a). The Pacific Decadal Oscillation. A brief overview for non - specialists, to appear in the Encyclopedia of Environmental Change. <http://www.atmos.washington.edu/ $\sim$ mantua/REPORTS/ PDO/PDO_egec.htm> [accedido: 09/07/2013]

Mantua, N.J. 1999 (b). The Pacific Decadal Oscillation and Climate Forecasting for North America. To appear in premiere issue of "Climate Risk Solutions" newsletter. <http://www.atmos.washington.edu/ - mantua/ REPORTS/PDO/PDO_cs.htm>[accedido: 09/07/2013]

Mantua N. J. \& S. R. Hare. 2002. The Pacific Decadal Oscillation. Journal of Oceanography (58): $35-44$. DOI 10.1023/A:1015820616384

McFarlane G.A. \& R.J. Beamish. 2001. The re-occurrence of sardines off British Columbia characterises the dynamic nature of regimes. Progress in Oceanography, $49(1-4)$ : 151 - 165. DOI: 10.1016/ S0079-6611(01)00020-9

Miller A.K. \& W.J. Sydeman. 2004. Rockfish response to low - frequency ocean climate change as revealed by the diet of a marine bird over multiple time scales. Mar Ecol. Prog. Ser. 281: 207 - 216.

Morón O. \& M. Sarmiento. 2001. Aspectos oceanográficos de El Niño 1997 - 98 y su relación con los recursos pelágicos. En: J. Tarazona, W.E. Arntz y E. Castillo de Maruenda (eds.), El Niño en América Latina: Impactos Biológicos y Sociales. Consejo Nacional de Ciencia y Tecnología. Lima, pp. 5 - 27.

Noakes D.J., R.J. Beamish \& M.L. Kent. 2000. On the decline of Pacific salmon and speculative links to salmon farming in British Columbia. Aquaculture 183(3-4): 363 - 386. doi:10.1016/S00448486(99)00294-X.

Noakes D.J. \& R.J. Beamish. 2009. Synchrony of Marine Fish Catches and Climate and Ocean Regime Shifts in the North Pacific Ocean. Marine and Coastal Fisheries: Dynamics, Management and Ecosystem Science1:155 - 168, 2009. DOI: 10.1577/C08 - 001.1.

Niquen M., A. Chipollini, A. Echevarría, J. Mori \& B. Buitrón. 2004. Cambios biológicos en los principales recursos marinos peruanos en el verano 2001. Inf. Inst. Mar. Perú - Callao 32 (1): 57 - 62.

Niquen M., M. Bouchon, D. Ulloa \& A. Medina. 2013. Análisis de la pesquería del jurel Trachurus murphyi en el Perú. En: Csirke J., R. GuevaraCarrasco, M. Espino (Eds.). Ecología, pesquería y conservación del jurel (Trachurus murphyi) en el Perú. Rev. peru. biol. número especial 20(1): 097- 106

Peterson W.T. \& F.B. Schwing. 2003. A new climate regime in northeast pacific ecosystems, Geophys. Res. Lett. 30(17), 1896, doi:10.1029/2003GL017528, 2003

Quinn W.H., V.T. Neal \& S.E. Antunez de Mayolo. 1987. El Niño Occurrences Over the Past Four and Half Centuries. Journal of Geophysical Research, 92 (C13):14449 - 14461. DOI 10.1029/JC092iC13p14449

QuinnT.J.,II. \& H.J. Niebauer.1995. Relation of eastern Bering Sea wallege pollack (Theragra chalcogramma) recruitment to environmental and oceanographic variables. Pp. 497 - 507. In R.J.Beamish, ed. Climate change and northern fish populations. Canadian Special Publications in Fisheries and Aquatic Sciences 121.
Samamé M., 1981. Las Pesquerías Demersales en el Perú. CPPS, Seminario Regional sobre los Recursos Demersales y su Medio Ambiente en el Pacífico Oriental. Lima, Perú 24-28 de noviembre de 1980. CPPS, Serie Seminarios y Estudios, 3, pp. 213-247.

Saitoh T., B. Cazelles, J. Olav Vik, H. Viljugrein \& N.Chr. Stenseth. 2006. Effects of regime shifts on the population dynamics of the grey sided vole in Hokkaido, Japan. Clim. Res.32:109 - 118 (2006), doi: $10.3354 / \mathrm{cr} 032109$.

Schwartzlose R.A., J. Alheit, A. Bakun, T. R. Baumgartner, R. Cloete, R.J.M. Crawford, W.J.Fletcher, Y. Green - Ruiz, E. Hagen, T. Kawasaki, D. Lluch - Belda, S.E. Lluch - Cota, A.D. MacCall, Y. Matsuura, M.O. Nevarez - Martínez, R.H. Parrish, C. Roy, R. Serra, K.V. Shust, M.N. Ward \& J.Z. Zuzunaga 1999. Worldwide large - scale fluctuations of sardina and anchovy populations. South African Journal of Marine Science 21:289 - 347. DOI: $10.2989 / 025776199784125962$

Swartzman G., A. Bertrand, M. Gutiérrez, S. Bertrand \& L. Vásquez. 2008. The relationship of anchovy and sardine to water masses in the Peruvian Humboldt Current System from 1983 - 2005. Prog. Oceanogr. 79: $228-237$.

Vert- pre K. A., R. O. Amoroso, O. P. Jensenc \& R. Hilborn. 2013. Frequency and intensity of productivity regime shifts in marine fish stocks. PNAS. January 29, 2013. 110. (5): 1779-1784

Wada T. \& L.D.Jacobson. 1998. Regimes and stock-recruitment relationships in Japanese sardine (Sardinops melanostictus), 1951 - 1995. Canadian Journal of Fisheries and Aquatic Sciences 55: 2455 - 2463.

Walters G.E. \& T.K.Wilderbuer. 2000. Decreasing length at age in a rapidly expanding population of northern rock sole in the Bearing Sea and its effect on managment advice. Netherlands Journal of Sea Research 44: 17 - 26

Wang C. \& J. Picaut. 2004. Understanding ENSO physics - A review, in: Earth's Climate: The Ocean-Atmosphere Interaction, edited by: C. Wang, S.P. Xie andJ.A. Carton., AGU Geophysical Monograph Series 147: $21-48$

Wang C., S.P. Xie \& J.A. Carton.2004. A global survey of oceanatmosphere interaction and climate variability, in: Earth's Climate: The Ocean - Atmosphere Interaction, edited by: C. Wang, S.P. Xie and J.A. Carton, AGU Geophysical Monograph Series 147: 1 - 19.

Wang C. \& P. C. Fiedler. 2006. ENSO variability and the eastern tropical Pacific: A review. Progress in Oceanography 69: 239 - 266.

Wilderbuer T.K., A.B. Hollowed, W.J. Ingraham, Jr., P.D. Spencer, M.E. Conners, N.A. Bond \& G.E. Walters. 2002. Flatfish recruitment response to decadal climatic variability and ocean conditions in the eastern Bering Sea. Progress in Oceanography 55: 235 - 247. DOI: $10.1016 /$ S0079-6611(02)00081-2

Wolter, K., 1987: The Southern Oscillation in surface circulation and climate over the tropical Atlantic, Eastern Pacific, and Indian Oceans as captured by cluster analysis. Journal of Climate and Applied Meteorology 26, 540 - 558. doi: http://dx.doi.org/10.1175/15200450(1987)026<0540:TSOISC $>2.0$. CO;2

Wolter, K., \& M.S. Timlin, 1993: Monitoring ENSO in COADS with a seasonally adjusted principal component index. Proc. of the 17th Climate Diagnostics Workshop, Norman, OK, NOAA/N MC/ CAC, NSSL, Oklahoma Clim. Survey, CIMMS and the School of Meteor., Univ. of Oklahoma, $52-57$.

Wolter, K., \& M.S. Timlin, 1998: Measuring the strength of ENSO - how does 1997/98 rank? Weather, 53, 315 - 324. DOI: $10.1002 /$ j.14778696.1998.tb06408.x

Wosnitza-Mendo C., R. Guevara-Carrasco \& M. Ballón. 2004. Possible causes of the drastic decline in mean length of Peruvian hake in 1992, vol. 21. Boletín Instituto del Mar del Perú. pp. 1 - 26.

Wyrtki K. 1967. Circulation and water masses in the Eastern Equatorial Pacific Ocean. Int. Jour. Oceanol. and Limnol. 1(2):117 - 147.

Zhuguo Ma. 2007. The interdecadal trend and shift of dry/wet over the central part of North China and their relationship to the Pacific Decadal Oscillation (PDO). Chinese Science Bulletin 52 (15): 2130 - 2139

Zuta S. \& O. Guillén. 1970. Oceanografía de las Aguas Costeras del Perú. Bol. Inst. Mar del Perú - Callao, 2 (5): 161 - 223. 